\title{
Em direção a Educação Ubíqua: aprender sempre, em qualquer lugar, com qualquer dispositivo
}

\author{
Débora Nice Ferrari Barbosa - UNILASALLE - nice@ unilasalle.edu.br \\ Dirléia Fanfa Sarmento - UNILASALLE - fanfa@ unilasalle.edu.br \\ Jorge Luis Victória Barbosa - PIPCA/UNISINOS - jbarbosa@ unisinos.br \\ Cláudio Fernando Resin Geyer - PPGC/UFRGS - geyer@ unilasalle.edu.br
}

\begin{abstract}
Resumo
A computação ubíqua visa atender pró-ativamente às necessidades dos usuários, atuando de forma invisível (background). Este cenário permite o desenvolvimento de ambientes voltados à educação ubíqua, onde a mobilidade do aprendiz e a percepção dos elementos que estão em seu entorno (contexto) são parte do processo educativo. Esse trabalho apresenta o GlobalEdu, uma infra-estrutura para suporte a processos educacionais direcionado à educação ubíqua. O sistema é composto de Módulos Educacionais e de um Agente Pedagógico, que acompanha o aprendiz, assistindo o processo educacional, independente do dispositivo de acesso. O modelo foi integrado ao ambiente ubíquo LOCAL e os resultados obtidos mostram a viabilidade da proposta.
\end{abstract}

Palavras-chaves - educação ubíqua, consciência do contexto, ambientes de educação ubíqua

\section{Towards Ubiquitous Learning: learning anywhere, any time with any device}

\begin{abstract}
This paper presents our experiences with Ubiquitous Learning. It also presents GlobalEdu, an architecture directed to ubiquitous learning. GlobalEdu allows continuous learning in a contextualized environment. The system is composed by Educational Modules and a Pedagogical Agent. It is implemented on top of an ubiquitous execution support environment. The Pedagogical Agent accompanies the learner, assisting his/her learning process, regardless of the user's device at the moment. The Educational Modules manage the environment's resources in order to provide learning processes. This paper also presents the integration between GlobalEdu and an execution support environment called LOCAL. Based on this integration, we created a prototype for a learning application.
\end{abstract}

Keywords - ubiquitous learning, context-awareness, ubiquitous learning environment

\section{Introdução}

Atualmente, a computação baseada em desktop, tradicionalmente estática, está emergindo para um paradigma altamente dinâmico. A mobilidade do usuário, portando dispositivos móveis e acessando recursos através das tecnologias de rede sem fio, traz a necessidade de prover um acesso uniforme e imediato às suas informações (Satyanarayanan, 2001). A Computação Ubíqua (Weiser, 1991) surge como um modelo V. 6 № 1, Julho, 2008 
computacional que visa atender pró-ativamente às necessidades dos usuários, atuando de forma invisível (background). O objetivo é prover uma integração contínua entre a tecnologia e o ambiente, auxiliando o usuário em suas tarefas cotidianas. Nesse modelo, as aplicações estão disponíveis independente de lugar e tempo. O usuário mantém o acesso à rede e à seu ambiente computacional, independente da sua localização física ou do dispositivo de acesso. Tecnologias da computação pervasiva (Satyanarayanan, 2001), consciência do contexto $^{1}$ (Dey, 1999) e de localização (Hightower, 2001) vem sendo utilizadas para suportar a computação ubíqua.

Recentemente, este cenário vem permitindo o desenvolvimento de ambientes para apoio à educação ubíqua (Chen, 2003). Nesse modelo, a mobilidade do aprendiz e a percepção dos elementos que estão em seu entorno (contexto) são parte do processo educativo, que pode ocorrer de forma contínua, global e transparente. Em direção a este cenário, algumas propostas estão em desenvolvimento ((Chen, 2003), (Ogata, 2003) e (Barbosa, 2005)). Esse trabalho apresenta o GlobalEdu, uma infra-estrutura para suporte a processos educacionais direcionado à educação ubíqua. O modelo possibilita a interação do aprendiz com os recursos e o ambiente ao seu redor, de forma contínua, através da percepção da sua mobilidade e de seu contexto. Esses aspectos são providos através do uso de informações do perfil do aprendiz, de objetos de aprendizagem e de elementos de contexto como instrumentos de apoio à educação.

Este artigo está organizado em seis seções: a seção dois apresenta a fundamentação tecnológica e pedagógica do trabalho. A seção três aborda o GlobalEdu e aspectos de sua arquitetura. Na quarta seção as decisões de desenvolvimento e validação da proposta são apresentados. Os trabalhos relacionados são abordados na seção cinco. Finalmente, a seção seis apresenta as conclusões.

\section{Em direção à educação ubíqua}

\subsection{Aspectos Tecnológicos}

Ambientes e experiências em direção a educação ubíqua integram diferentes aspectos tecnológicos. Em função disso, surgem modelos educacionais como: aprendizagem com mobilidade (Roschelle, 2002), aprendizagem baseada na localização (Price, 2004), educação consciente do contexto ((Toivonen, 2003), (Barbosa, 2006)), educação pervasiva (Coatta, 2003) e, finalmente, modelos que integram esses aspectos educação ubíqua (Ogata, 2003), (Rogers, 2005).

Ogata (2003) apresenta diferenciações entre alguns desses modelos, estabelecendo uma comparação entre ambientes educacionais baseados em desktop até ubíquos, analisando o nível de integração ao ambiente (embeddedness) e à mobilidade. Segundo Ogata, a educação baseada em desktop possui um baixo nível de mobilidade e de integração com o ambiente real, não permitindo transparência para o usuário. Na aprendizagem com mobilidade, os aprendizes têm a capacidade de se mover fisicamente utilizando recursos e acessando informação (Roschelle, 2002). Entretanto, os dispositivos não possuem flexibilidade para obter informações sobre o contexto em que

\footnotetext{
${ }^{1}$ Segundo Dey (1999) “contexto é qualquer informação que pode ser usada para caracterizar a situação de uma entidade. Uma entidade é uma pessoa, um lugar ou um objeto que é considerado relevante para a interação entre o usuário e uma aplicação, incluindo o próprio usuário e a própria aplicação.” [grifo do autor]
}

V. 6 № 1, Julho, 2008 
ele está inserido. Essa característica é presente em sistemas de educação pervasiva através de dispositivos (sensores) que são integrados ao ambiente e se comunicam mutuamente. Em função disso, a mobilidade é restrita, dependente da localização do aprendiz. Em uma aplicação educacional consciente do contexto, os elementos do contexto (localização do aprendiz, dispositivos, atividades, recursos, etc) devem ser considerados como integrantes do processo educativo, auxiliando o aprendiz na sua própria aprendizagem e na percepção dos elementos que estão a sua volta. Um sistema de educação ubíqua provê alto nível de mobilidade e integração com os elementos do ambiente, suportando a consciência do contexto, da localização e da mobilidade do aprendiz, de forma contínua e integrada.

Para o GlobalEdu, educação ubíqua é um processo que pode ocorrer em qualquer tempo e lugar, de forma adaptada, contínua e integrada ao cotidiano do aprendiz (Barbosa, 2005). No cenário da educação ubíqua, novos pressupostos educacionais devem ser pensados, uma vez que os recursos pedagógicos podem ser acessados a qualquer momento e em qualquer lugar. $\mathrm{O}$ suporte ubíquo permite a construção de programas de aprendizagem relacionados com questões dinâmicas do contexto do aprendiz. O ambiente controla as aplicações orientadas à educação, possibilitando que o contexto seja vinculado com os objetivos pedagógicos. A educação neste cenário é dinâmica e os recursos educacionais estão distribuídos em contextos. Baseado nos objetivos do aprendiz, o sistema pode gerar intervenções do tipo: um material/pessoa/dispositivo que se relaciona com seu objetivo está disponível para você agora (contexto). Dessa forma, percebe-se que a essência da educação ubíqua está em perceber que a informação está presente no dia-a-dia das mais diferentes formas e em diferentes locais, relacionando com os processos educativos do aprendiz. A tecnologia que suporta a educação ubíqua deve prover estes aspectos através de mecanismos que permitem conhecer o aprendiz, o contexto que o envolve e como ele se relaciona com esse contexto, adaptando com isto processos e recursos educacionais.

A consciência de contexto é um modelo computacional importante nesse cenário. No apoio à educação, a consciência de contexto permite relacionar informações explicitas referentes ao aprendiz, tais como suas preferências e objetivos, com informações implícitas decorrentes a sua localização física. Desta forma, é possível que uma aplicação indique para o aprendiz, de maneira pró-ativa, um determinado evento ou recurso de seu interesse disponível no contexto onde ele se encontra. Nesta perspectiva, os ambientes virtuais de educação ubíqua devem possibilitar processos educacionais relacionados com a situação que envolve o aprendiz (consciência do contexto).

\subsection{Aspectos Pedagógicos}

Vivemos na era do conhecimento e, em decorrência, emerge uma nova cultura de aprendizagem (Pozo, 1996) em que as dimensões espaço-temporais precisam ser revistas. Ou seja, com a contribuição do desenvolvimento tecnológico, urge pensar que a aprendizagem não se restringe e tampouco se efetiva somente dentro das instituições educativas. É necessário romper com esta visão fragmentada, localizada e linear do ato de aprender e partir para uma compreensão fundamentada na idéia de rede, em que o sujeito pode aprender em diferentes espaços educativos, em qualquer tempo e espaço, via mediação tecnológica. Vygotsky $(1991,1995,1998)$ defende a idéia de que a relação homem-mundo não é uma relação direta, mas sim é uma relação mediada principalmente por ferramentas (instrumentos) e signos, que auxiliam a atividade humana. Conforme Oliveira (1993, p. 26) a mediação pode ser compreendida como o 
"[...] o processo de intervenção de um elemento intermediário numa relação; a relação deixa, então, de ser direta e passa a ser mediada por esse elemento" [grifo da autora].

Considerando as tecnologias da informação e da comunicação, enquanto instrumento, entende-se que o aprendiz necessita desenvolver uma série de competências de forma a beneficiar-se e se apropriar dessas tecnologias. Nesse sentido, considera-se um sujeito cognitivo que se constitui e constrói seu conhecimento através das ações que exerce sobre o meio circundante. Este sujeito possui uma dimensão ativa, isto é, são as ações que possibilitam ao sujeito transformar a si mesmo e transformar o objeto, e neste continuum ir se desenvolvendo e construindo novas estruturas de conhecimento (Piaget, 1976).

Esta idéia de sermos sujeitos inacabados, sinaliza para um vir a ser em que a formação humana é vista como um processo contínuo. A pessoa, enquanto construtora de si mesmo, precisa estar aberta para buscar novas aprendizagens e ampliar suas experiências e conhecimentos. Para tanto, a idéia de um sujeito autônomo cognitivamente é fundamental, pois a aprendizagem e a formação contínua é condição sine qua non numa sociedade do conhecimento. As tecnologias oferecem recursos para uma educação centrada no aprendente, ou seja, ele próprio, pode se tornar um gestor do seu conhecimento. Tais recursos facilitam o acesso a diferentes fontes de informação permitindo que o sujeito trace suas metas e rotas de aprendizagem considerando seu estilo cognitivo, conhecimentos prévios, interesses, necessidades, competências, recursos disponíveis e a sua realidade sócio-cultural e econômica. Portanto, o desenvolvimento de um indivíduo autônomo, e a possibilidade que ele aprenda de forma significativa (Ausubel, 1983 apud Moreira, 1999), deve ser o elemento central dos processos educacionais mediados e potencializados pelas tecnologias. Com isso, os ambientes virtuais de educação devem propiciar o desenvolvimento de indivíduos capazes de definir recursos pedagógicos significativos para sua aprendizagem e interagir com outros indivíduos, de forma a construir seu conhecimento. Esta concepção é fundamental na educação em um cenário ubíquo. Esse é dinâmico, possui uma variedade de recursos disponíveis e a conectividade pode ser global.

Nesse cenário, os aprendizes devem definir seus próprios objetivos educacionais e, como ser autônomo, cabe a ele a organização do que aprender. Além disso, a aprendizagem não ocorre somente na sala de aula, mas está presente no dia a dia do aprendiz. A sala de aula é um dos espaços. O contexto que envolve o aprendiz também possui elementos de aprendizagem significativos e que devem ser explorados. Desta forma, sistemas de suporte à educação ubíqua devem perceber o aprendiz no virtual, como também o perceber integrado ao seu meio (contexto), sendo ele também parte do meio.

\section{Uma proposta para suporte à educação ubíqua}

O GlobalEdu é uma infra-estrutura para suporte a processos educacionais direcionado à educação ubíqua. $\mathrm{O}$ modelo possibilita a interação do aprendiz com os recursos e o ambiente ao seu redor, de forma contínua, através da percepção da sua mobilidade e de seu contexto. Esses aspectos são providos através do uso de informações do perfil do aprendiz, de objetos de aprendizagem e de elementos de contexto como instrumentos de apoio à educação. A arquitetura é organizada em camadas (Figura 1). A Camada de Aplicação (CA) contém o Agente Pedagógico (AP).

V. 6 № 1, Julho, 2008 
A Camada de Sistema (CS) contém os Módulos Educacionais, que manipulam o contexto do aprendiz, seu perfil e conteúdos, além de Módulos de Suporte que auxiliam na execução do AP e dos recursos que ele necessita. A Camada de Execução (CE) compreende o ambiente (middleware) de execução.

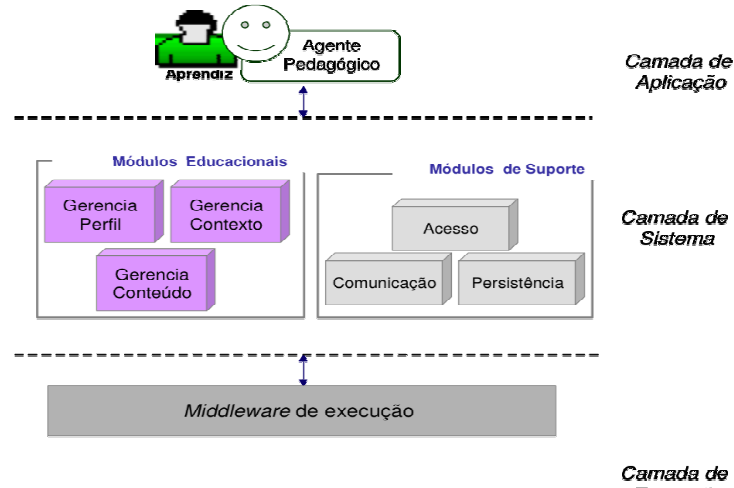

Figura 1. Visão geral da arquitetura GlobalEdu

\subsection{Camada de Aplicação - CA}

A CA contém o Agente Pedagógico (AP) que auxilia o aprendiz no ambiente ubíquo, de forma adaptada e contínua, tendo como objetivo servir de interface entre o aprendiz e o ambiente, tornando o processo educacional algo natural. São características principais do AP: (a) Mobilidade - tem a capacidade de mover-se para os dispositivos utilizados pelo aprendiz, adaptando-se ao dispositivo; (b) Sempre presente - uma vez instanciado no dispositivo do aprendiz, se mantém operando mesmo quando está desconectado da rede, com restrição de acesso aos demais módulos do ambiente; (c) Consciência do contexto - com o auxílio dos Módulos Educacionais, apresenta ao aprendiz informações de acordo com suas necessidades e o local em que ele está, relacionando contexto e recursos conforme seus objetivos e interesses.

\subsection{Camada de Sistemas - CS}

Os Módulos Educacionais (ME) são responsáveis pelo armazenamento e gerenciamento das informações educacionais manipuladas no GlobalEdu, executando tarefas em dispositivos estáveis da arquitetura. O ME Gerencia Perfil manipula o perfil do aprendiz, gerando e fornecendo essa informação aos demais módulos da arquitetura (Figura 2). Os padrões PAPI (2001) e LIP (2001), bem como informações referentes ao estilo de aprendizagem (Felder, 1998) do aprendiz, suas atividades e seu histórico no ambiente são usados para compor o perfil. O módulo atualiza os demais módulos do sistema sempre que as informações forem alteradas. Isso ocorre quando identificado uma incoerência no estilo de aprendizagem, um determinado objetivo foi alcançado ou quando alterações nas informações forem detectadas. 


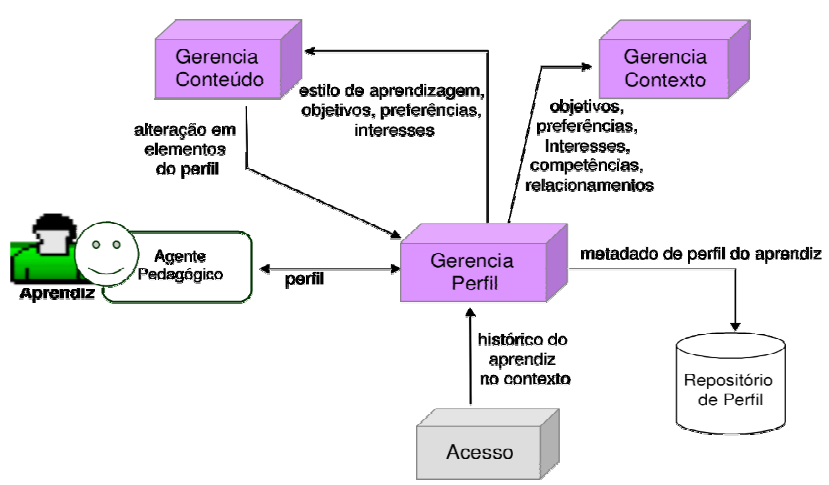

Figura 2. Integração do módulo Gerencia Perfil no GlobalEdu

O ME Gerencia Contexto controla informações do contexto de interesse do aprendiz e da adaptação dos recursos que ele está manipulando, gerenciando aspectos de contexto Social e Físico do aprendiz (Figura 3). O Contexto Social descreve elementos estáticos e dinâmicos de uma localização caracterizada como Região_geográfica (ex., campus universitário), ou Sub_região (ex., uma Biblioteca). Os elementos estáticos descrevem informações associadas a pessoas, eventos e recursos de uma localização (Contexto Localização). Quando o aprendiz entra em uma localização, o módulo adapta e sugere acesso às informações que são relevantes para ele, disponíveis através de um Repositório de Contexto Localização descrito em XML. O elemento dinâmico refere-se à presença de outros aprendizes no contexto. Com isso, o sistema os relaciona, procurando identificar preferências, objetivos e competências em comum. O relacionamento pode ser: (a) Por semelhança - interesses e/ou objetivos em comum; (b) Por complementaridade - objetivos semelhantes às competências de outros; e (c) Por relacionamento anterior - relacionamentos já ocorridos em um contexto. O Contexto Físico manipula informações de interesse da execução do AP e dos recursos educacionais acessados pelo aprendiz, quais sejam: tipo e banda da rede que o aprendiz está acessando, sua localização, o tipo e a autonomia do dispositivo em uso.

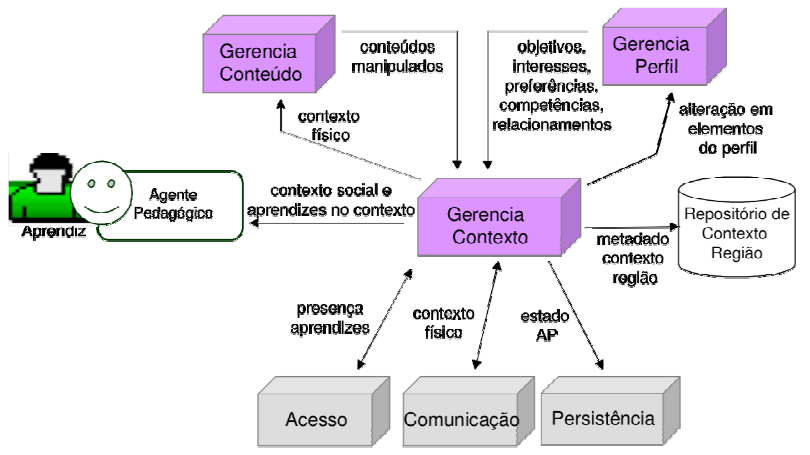

Figura 3. Interações do módulo Gerencia Contexto no GlobalEdu

O conteúdo no GlobalEdu é manipulado como objetos de aprendizagem, representados através do padrão IEEE/LOM (2002). O ME Gerencia Conteúdo gerencia e disponibiliza objetos de aprendizagem conforme o perfil e contexto do aprendiz. Para isso, o módulo manipula os Repositórios de Objetos de Aprendizagem da arquitetura e obtém o perfil do aprendiz e seu contexto (Figura 4). Alterações no perfil 
ou no contexto físico, como banda de rede e dispositivo, fazem com que o componente adapte a apresentação do objeto educacional, enviando um novo conteúdo ao AP.

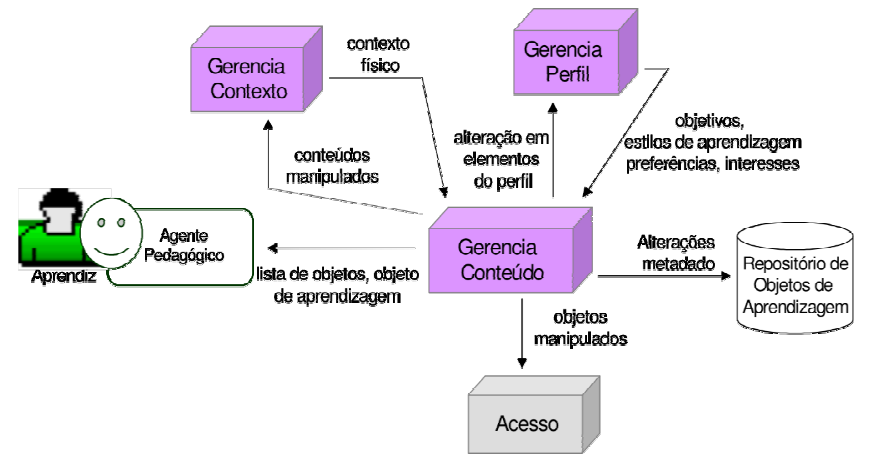

Figura 4. Integração do módulo Gerencia Conteúdo no GlobalEdu

Os Módulos de Suporte são responsáveis pelos elementos que auxiliam a execução do AP e dos Módulos Educacionais. São os seguintes: (1) Acesso - é responsável por registrar e controlar o acesso do aprendiz no GlobalEdu. Também gera seu histórico de mobilidade (localizações, dispositivos usados, recursos e programas executados. (2) Persistência - armazena o estado do AP e dos demais dados sobre o aprendiz, gerenciados pelos demais componentes da arquitetura. (3) Comunicação garante a abstração entre os elementos do ambiente e o sistema de execução.

\section{Avaliação do sistema}

O GlobalEdu está sendo integrado ao ambiente de execução LOCAL (Barbosa, 2006). Este é formado por quatro componentes: um sistema de perfis, um sistema de localização, um sistema de envio de mensagens contextuais e um assistente pedagógico. O Sistema de Perfis foi adequado seguindo alguns elementos especificados no modelo do GlobalEdu. O assistente pedagógico corresponde a uma versão simplificada do AP. Foram incorporados na arquitetura dois elementos que envolvem protótipos dos Módulos Educacionais: um repositório de objetos de aprendizagem e um tutor. $\mathrm{O}$ primeiro armazena e indexa o conteúdo (Gerencia Conteúdo). O segundo integra e simplifica as ações realizadas pelos módulos Gerencia Contexto e Gerencia Perfil. O Tutor usa os perfis e as informações de contexto, mais especificamente localização, para inferência de oportunidades de ensino e de aprendizagem.

O sistema foi avaliado junto ao curso de Engenharia da Computação da Unisinos. A infra-estrutura de rede sem fio do prédio do curso é composta de quatro antenas wireless Cisco Aironet distribuídas no andar. $\mathrm{Na}$ avaliação foram utilizados como dispositivos móveis iPAQs 4700 disponíveis no MobiLab ${ }^{1}$. A população amostra consistiu em vinte sujeitos, entre alunos e professores do curso. Primeiramente, o objetivo do estudo, bem como características básicas do sistema foram apresentadas. Após, os sujeitos inseriram seus dados pessoais no AP e utilizaram o sistema. Ao término do uso, preencheram um questionário, permitindo a análise de dados e a avaliação do sistema.

\footnotetext{
${ }^{1}$ http://www.inf.unisinos.br/ mobilab V. 6 № 1 , Julho, 2008
} 
Algumas considerações podem ser destacadas a partir da análise de cada questão do questionário. Os aspectos de consciência do contexto propostos no GlobalEdu, bem como a avaliação do protótipo, foram analisados em conjunto (Figura 5). As questões (2 a 8) abordam informações sobre pessoas, eventos e recursos disponíveis no contexto, relacionamento (complementaridade/similaridade) com os outros usuários do contexto, apresentação do conteúdo relacionado a um evento ocorrendo no mesmo local, além do uso do sistema para indicar a localização do usuário. Conforme pode ser observado, os resultados indicam a boa aceitação quanto aos elementos de contexto apresentados, visto que os mesmos consideraram o sistema bom e excelente quanto às informações de contexto. Percebe-se, também, que um pequeno percentual da amostra considerou o sistema satisfatório, não incidindo de forma negativa na avaliação.

As questões 9 a 11 do questionário avaliaram o uso de sistema no dia-a-dia e como apoio ao aprendizado em sala de aula, além da interação com os elementos presentes nos diversos contextos aos quais o aprendiz se move. A Figura 6 apresenta o resultado das avaliações para essas questões. Os resultados indicam a boa aceitação dos sujeitos quanto aos aspectos avaliados. Mais de $60 \%$ da amostra consideram o sistema bom ou excelente quanto aos aspectos avaliados. Os $32 \%$ da amostra que consideraram o sistema satisfatório também representam um resultado satisfatório, visto que a avaliação foi realizada dentro de um cenário limitado e controlado (dentro de um prédio e considerando como contexto a localização). Percebe-se, com isso, uma boa indicação para o uso diário do sistema pelos usuários (uso em vários contextos e de forma contínua).

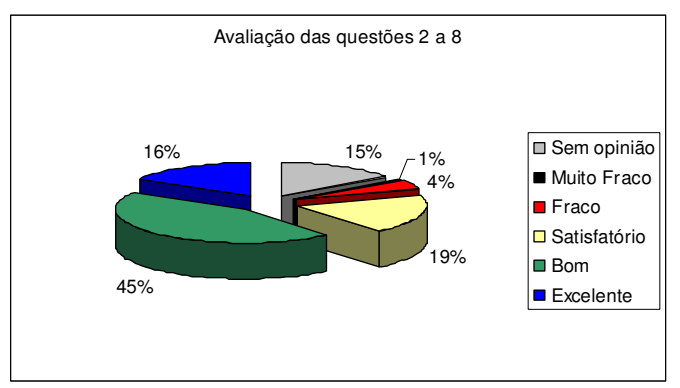

Figura 5: Análise das avaliações quanto às informações de contexto

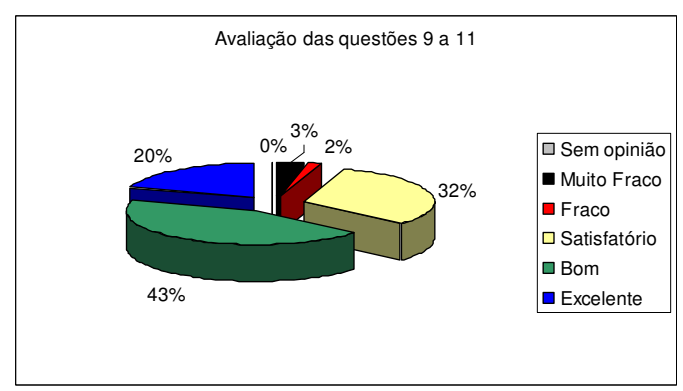

Figura 6: Análise das avaliações quanto aos objetivos do GlobalEdu

A questão 1 refere-se a adaptação da interface do sistema ao dispositivo de acesso. Embora o sistema tenha sido utilizado somente com iPAQs, o AP possui interface adaptada para tablet PC e desktop. Essa funcionalidade foi apresentada aos sujeitos, recebendo uma boa avaliação quanto a esse aspecto (Figura 7). A questão 9 explorou à interação com os usuários presentes em uma localização (Figura 8), onde 95\% dos sujeitos consideram esse aspecto entre satisfatório e excelente. Desse percentual, $60 \%$ analisou essa característica como boa. Considerando o número de sujeitos da amostra, bem como suas habilidades em computação e experiência com outros tipos de interação mediada por computador, considera-se esse resultado satisfatório. 


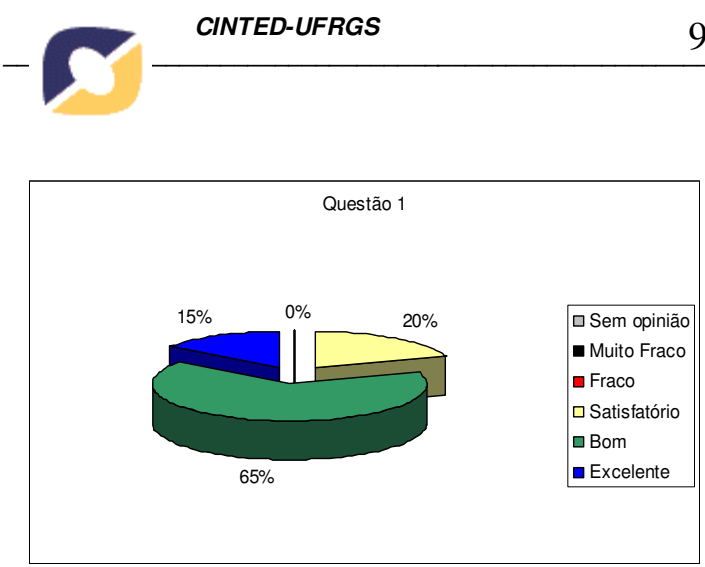

Figura 7: Avaliação da adaptação à interface

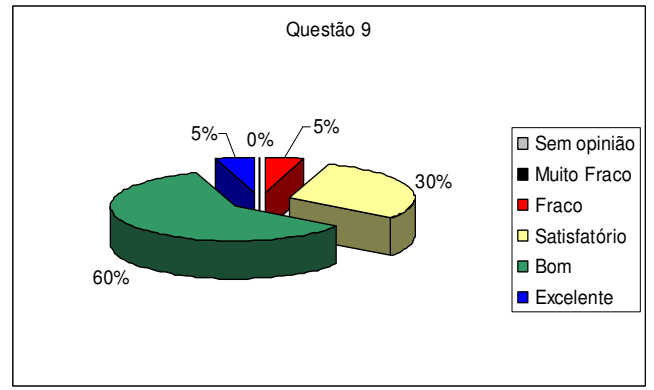

Figura 8: Avaliação quanto à interação com outros usuários em uma localização

\section{Trabalhos relacionados}

Elena (Simon, 2003) e SeLeNe (2004) permitem que o aprendiz acesse uma rede de aprendizagem via Web e obtenha objetos de aprendizagem personalizados, mas não consideram a mobilidade do aprendiz e elementos do contexto. Ogata (2003) apresenta o sistema CLUE (Collaborative Learning support system with an Ubiquitous Environment) que permite colaboração em um contexto ubíquo local, voltado para o domínio específico de ensino da língua japonesa. Rogers (2005) não usa perfis, somente localização. A integração de consciência do contexto, da mobilidade e do perfil do aprendiz em um ambiente com suporte a elementos da computação ubíqua é a principal contribuição do GlobalEdu. O AP acompanha e auxilia o aprendiz sempre que este está presente no ambiente e não possui um foco específico de atuação.

\section{Conclusões}

Este trabalho tem como principal contribuição a apresentação do GlobalEdu, uma infra-estrutura para suporte a processos educacionais direcionado à educação ubíqua. O modelo possibilita a interação do aprendiz com os recursos e o ambiente ao seu redor, de forma contínua, através da percepção da sua mobilidade e de seu contexto. A integração da proposta com o ambiente LOCAL foi apresentada. A avaliação preliminar com o sistema mostrou que sistemas educacionais ubíquos podem melhorar a interatividade do aprendiz nos diversos contextos em que ele se move. Uma vez que o sistema percebe o aprendiz tanto no virtual como no real (contexto), é possível auxiliar o aprendiz a relacionar os conhecimentos com sua realidade, criando seus próprios caminhos de aprendizagem, em interação com o meio. Esse aspectos pressupõem um sujeito autônomo, responsável pela construção do seu conhecimento e de uma aprendizagem significativa. Atualmente, novos componentes da arquitetura GlobalEdu estão sendo desenvolvidos e integrados ao ambiente LOCAL, tais como funcionalidades do AP que permitem ao aprendiz relacionar-se com seu contexto social e manipular objetos de aprendizagem no sistema.

\section{Referências}

BARBOSA, D. N. F. et al. GlobalEdu - an architecture to support learning in a Pervasive Computing Environment. In: IFIP TC10 Working Conference: EduTech 2005, 2005, Perth. New York : Springer, 2005. p. 1-10 
BARBOSA, J. L. V. et al. LOCAL: Um Modelo para Suporte à Aprendizagem Consciente de Contexto. In: XVII Simpósio Brasileiro de Informática na Educação, 2006, Brasília. SBIE. Porto Alegre : SBC, 2006. p. 437-446.

CHEN, H. et al. Semantic Web in a Pervasive Context-Aware Architecture. Artificial inteligente in Mobile System. In UBICOMP, 2003. October, Seattle.

COATTA, T.; KAUFMANN, R. A new plataform for pervasive learning. In: World Conference on Educational Multimedia, Hypermedia and Telecommunication, 2003.

DEY, A. K. et al. Towards a better understanding of context and context-awareness. TR GIT-GVU-99-22, Georgia Institute of Technology, June, 1999.

FELDER, R.M and L.K. SILVERMAN. Learning and Teaching Styles in Engineering Education, In Engineering Education, 78(7), 1998, 674.

HIGHTOWE, J.; BORRIELLO, G. "A Survey and Taxonomy of Location Systems for Ubiquitous Computing”, Computer, 34(8), Ago, 2001, pp.57-66

IEEE/LTSC. Learning Technology standards committee. Disponível em: http://ieeeltsc.org/. Acesso em março, 2008.

LIP. Learner Information Package Specification 1.0. 2001. http://www.imsglobal.org/profiles/index.html. Acesso em março, 2008.

MOREIRA, M. A. Aprendizagem significativa. Brasília: Editora Universidade de Brasília. 1999. 129p.

OGATA, H., YANO, Y. How Ubiquitous Computing can support language learning. Proc. of KEST, 2003, p.1-6.

PAPI. IEEE P1484.2/d7, 2001. Public and Private information for learners. http://jtc1sc36.org/doc/36N0179.pdf. Acesso em março, 2008.

PIAGET, Jean. Psicologia e pedagogia. 4ed. Rio de Janeiro: Forense, 1976.

PRICE, S.; ROGERS, Y. Let's get physical: the learning benefits of interacting in digitally augmented spaces. Computers \& Education, n.43, 2004, 137-151.

POZO, Juan I. Aprendices y Maestros. Editorial Alianza. Madrid. 1996.

ROGERS, Y; et al. Ubi-learning Integrates Indoor and Outdoor Experiences. Communications of the ACM, v. 48, n. 1, p.55-59, January, 2005

ROSCHELLE, J.; ROY P. A walk on the WILD side: How wireless handhelds may change computer-supported collaborative learning. In: International Conference on Computer-Supported Collaborative Learning, Colorado, 2002, January 7-11.

SeLeNe. Self e-Learning Networks. 2004. http://www.dcs.bbk.ac.uk/selene/. Acesso em Março, 2008.

SATYANARAYANAN, M. Pervasive Computing: Vision and Challenges. IEEE Personal Comunications, New York, v.4, n.8, Aug, 2001.

SIMON, B.; et al. Elena: A Mediation Infrastructure for Educational Services. Proc. of WWW Conference. Budapest, Hungary, May, 2003.

TOIVEN, S. et al. Facilitating Mobile Users with Contextualized Content. In: Artificial Intelligence in Mobile System Workshop 2003. Proceedings... Seattle, Oct., 2003.

VIGOTSKY, Lev S. A formação social da mente. (Orgs. Cole et al.) 4. ed. São Paulo: Martins Fontes, 1991.

VYGOTSKY, Lev S. Historia del desarrollo de las funciones psíquicas superiores. Obras Escolhidas III: Problemas del desarrollo de la psique. Madrid: Centro de Publicaciones del M.E.C y Visor Distribuiciones, 1995. 
VIGOTSKY, Liev S. El problema del desarrollo cultural del niño. IN: BLANCK, Guillermo. (Comp.). El problema del desarrollo cultural del niño y otros textos inéditos. Buenos Aires: Editorial Amagesto, 1998, p. 31-55.

YAMIN, A. C. et al. ISAM: a Pervasive View in Distributed Mobile Computing. In: IFIP/IEEE Network Control and Engineering (NET-CON'2002), 2002, Paris. New York: IEEE Press.

WEISER, Mark. The Computer for the 21st Century. Scientifc America, set., 1991, p. 94-104; reimpresso na IEEE Pervasive Computing, New York, v. 1, n. 3, sep. 2002. 